\title{
Pregnant and postpartum women with atypical glandular Cells: Follow-up and Evaluation for high-risk HPV
}

\author{
Intisar Salim Pity \\ Dept of Pathology, Faculty of Medical Science, UOD, Duhok, Iraq \\ Email address: \\ intisarsalimpity@gmail.com
}

\section{To cite this article:}

Intisar Salim Pity. Pregnant and Postpartum Women with Atypical Glandular Cells: Follow-up and Evaluation for High-Risk HPV. Cancer Research Journal. Vol. 1, No. 4, 2013, pp. 31-36. doi: 10.11648/j.crj.20130104.11

\begin{abstract}
Objectives: The study was an attempt: to find out the prevalence of atypical glandular cells of undetermined significance (AGC) in cervicovaginal smears of pregnant and postpartum women in Duhok-Iraq; follow up these women to investigate the underlying pathology and to test for high-risk HPV (HR-HPV). Methods: From May 2005 to June 2013, all Pap smears of pregnant and postpartum women were analyzed and women with AGC cytology were enrolled in the study. One hundred and fifty two women, for whom follow up was possible, were subjected to colposcopic directed cytology and/or histology for at least 48 months from the initial reading. Their results were comprehensively evaluated, and HR-HPV DNA testing was performed by conventional PCR in 56 cases. Results: Overall, AGC cytology formed $6.7 \%$ of all pregnant and postpartum women's Pap smears and 29.6\% were at risk for neoplastic epithelial lesions (AGC and premalignant lesions). The diagnosis of $\geq$ low squamous intraepithelial lesion (LSIL) was observed in $14.5 \%$ of cases among whom significantly high frequency of HR-HPV DNA was noted compared with those having persistent AGC or negative atypia (NILM). Conclusions: Identical to non-pregnant women, pregnant and postpartum women with AGC cytology are at risk of harboring premalignant and malignant (squamous or glandular) lesions, and testing of AGC cytologic specimens for HR-HPV might help predict neoplastic cases but not to distinguish malignant from premalignant lesions.
\end{abstract}

Keywords: Pap smear, AGC, Follow Up, High-risk HPV-DNA

\section{Introduction}

The term "atypical glandular cells of undetermined significance (AGC or AGUS)" is one of the diagnostic Pap smear categories reported in the Bethesda system 2001.1 This category is defined as glandular cells demonstrating nuclear atypia that exceeds reactive or reparative changes but lacks unequivocal features of adenocarcinoma.1,2 While AGC cytology results represent only a small percentage of Pap tests and majority of women have trivial lesions on subsequent follow up, a significant percentage represents a corresponding high-grade lesion that warrant either closer surveillance or aggressive intervention.3,4,5,6,7

Pregnancy is an excellent opportunity for detection of preneoplastic lesions and tumors at early stages because of eversion of transitional zone during this period.7 Identifying AGC during pregnancy and postpartum period is particularly difficult because of the confusion with cells due to the physiological changes occurring during this period (hyperplasia of the glandular epithelium, presence of decidual and trophoblastic cells in addition to Arias-Stella reaction).
Another important event is the eversion of endocervical epithelium during pregnancy which may remain in the first few weeks after delivery. This event causes the transformation zone to be more exposed to physical trauma, infections and vaginal $\mathrm{pH}$; all these elements generate reparative changes that may be misinterpreted as AGC in cervicovaginal smears.7

Evaluation and management of AGC Pap smears often prove to be perplexing and difficult for the physicians. One of the challenges in managing this condition is that the neoplastic lesion is often located at area that is difficult to sample such as high endocervical canal, endometrium or occasionally adnexa.5,8 While the guidelines have been established for management of AGC in non-pregnant patients, special considerations are required when this is discovered during pregnancy. Management options of minor cytologic changes are similar to those described in non-pregnant patients except that endometrial sampling and endocervical curettage are unacceptable because of the risk of disrupting the pregnancy. As well, conization in pregnancy is often associated with high rates of complications 
and thus should be reserved for patients with suspected invasive cancer. On the other hand, colposcopy is preferred during pregnancy.5,6,7,9

Several articles have dealt with the issues of the natural history of atypical squamous cells of undetermined significance (ASC-US), atypical squamous cells cannot exclude high squamous intraepithelial lesion (ASC-H) and AGC cytology.4-6,10-13 However to our best knowledge, little data are available from developing countries considering the long term follow up consequences of AGC Pap smears during pregnancy and postpartum period (within six weeks after delivery), and Iraq is one country that lacks the data related to the AGC cytology. Thus the current study, in parallel with others that could be done in the same discipline might provide an insight on the clinical significance of AGC Pap smear among pregnant and postpartum women in this area of the world.

To find the prevalence of AGC among pregnant and postpartum women, we studied Pap smears at these periods from May 2005 to June 2013; to determine the long-term outcome of these women, we followed them for a minimum of 48 months and their subsequent cytologic and histologic results were comprehensively evaluated, in addition to testing those with risky findings for high-risk HPV (HR-HPV).

\section{Methods}

During a period extended from May 2005 to June 2013, the study was conducted in Duhok Medical Laboratories and Scientific Research Center, Duhok-Iraq. Ethical approval was obtained from the Ethics Committee at the Faculty of Medicine, University of Duhok. After informed consent was obtained, cervical smears were collected from 2607 pregnant and postpartum women using a wooden Ayre's spatula. Smears were directly fixed by $95 \%$ ethanol then stained with Hematoxylin and Eosin (H\&E) stains or with the conventional Papanicolaou stain. Slides showing AGC cytology were initially reevaluated and further scrutinized by an experienced cytopathologist (IS Pity) to conform eligibility to the study. When the diagnosis of AGC was confirmed, an attempt was made to keep patients under follow up at 3-6 months interval for at least 48 months. One hundred and fifty two women, for whom follow up was possible, were recruited in the study. Cohorts were referred for colposcopic examination, using aqueous solution of $5 \%$ glacial acetic acid and lugols' solution. Subsequent follow up depended on the last Pap reading and whether the woman was pregnant or postpartum. All postpartum women with persistent abnormal Pap smears were subjected to colposcopic directed punch biopsy using Gaylor-Medina forceps. Other modes of histologic samplings included cervical conization, endocervical curettage, endometrial sampling, removal of polyp and total hysterectomy. Majority of patients were treated with more than one procedure. Pregnant ladies with persistent abnormal Pap reading were followed up by Pap smearing at 3 months intervals until conception, thence they were dealt with as postpartum women.

Tissue specimens were fixed in $10 \%$ buffered formalin then paraffin embedded, $4 \mu \mathrm{m}$ thick sections cut and processed routinely for $\mathrm{H} \& \mathrm{E}$ staining. Cytologic and histologic results were classified and reported, as previously described as follows: Negative for intraepithelial lesion or malignancy (NILM) for negative pathology, squamous metaplasia, reactive changes or inflammatory lesions; AGC for equivocal cases where the changes were suggestive of but inadequate for a definitive interpretation of adenocarcinoma in situ (AIS) or adenocarcinoma; low squamous intraepithelial lesion (LSIL) for koilocytotic atypia and cervical intraepithelial neoplasia type-1 (CIN I); and high squamous intraepithelial lesion (HSIL) for CIN II and CIN III. Severely dysplastic glandular epithelium was put under AIS and when invasion was definite, the diagnosis of squamous cell carcinoma or adenocarcinoma carcinoma was given accordingly.10 All specimens were reviewed again, and the least favorable histologic or cytologic result was considered as the final diagnosis.

High-risk human papillomavirus genomic DNA was tested on 56 samples. These cases included all patients showing carcinoma $(n=2)$, premalignant lesions $(n=20)$, cases with persistent AGC $(n=15)$ and those with cervical microglandular hyperplasia $(n=4)$, in addition to randomly selected samples from NILM, endometrial polyps and endocervical polyps, 5 cases each. Crude extracts of the cervical scrapes were used, and samples were collected in phosphate buffer saline solution. The genomic DNA was extracted by the method given by the manufactures'. High-risk HPV (HR-HPV) DNA analysis protocol was performed by the conventional PCR-based assay using a PCR-mix kit provided by Sacace Biotechnology (Sacace Company, Italy, and Catalog No. V-26-50R). The kit contained primers directed against $13 \mathrm{HR}-\operatorname{HPV}(16,18,31,33$, $35,39,45,51,52,56,58,59$, and 68). PCR technique and interpretations were carried out according to the recommendations supplied by the manufacturers' (Sacace Company, Italy, and Catalog No. V-26-50R) and as described previously.16,17 Negative control (DNA buffer) and positive controls (HPV type 31, 39 and 56) were run with the technique.

Interpretations of cytology, histology and HR-HPV testing were performed separately and blinded to the other test findings. Results were then analyzed by comparing cytological and histological criteria and HPV profile.

Statistical analysis: Descriptive statistics were used to summarize demographic variables. Data were collected and analyzed on the Statistical Package for Social Sciences (SPSS/PC+) and Chi-squared test was used to evaluate the associations between the risky readings and HR-HPV. The p-value of equal or less than 0.05 was considered as statistically significant.

\section{Results}

Out of 2607 pregnant and postpartum women, 174 (6.7\%) 
women had AGC reading "Fig. 1". Patients' ages ranged from 23 to 43 years (mean: 31.8 years). A wide spectrum of atypical glandular cell morphology was observed in cervical smears of our series "Fig. 2-5".

Table 1 demonstrates the detailed follow up findings of AGC Pap smears which revealed NILM in 63 (41.5\%) cases. Twenty five $(16.5 \%)$ cases remained equivocal on follow up cytology and histology and hence reported as AGC. Premalignant lesions were demonstrated in 20 (13.2\%) cases (6 LSIL, 8 HSIL and 6 AIS). Thus cases at risk for epithelial lesions (AGC and premalignant lesions) accounted for $29.6 \%$ of cases $(n=45)$. Malignancy was encountered in 2 cases (well differentiated endometrial adenocarcinoma and well differentiated squamous cell carcinoma). The diagnosis $\geq$ LSIL was encountered in 22 (14.5\%) cases. A concomitant ASC-US (ASC-US/AGC) was reported at initial smears in 7 cases. On follow up these cases turned to be LSIL ( $n=1)$, HSIL $(n=5)$ and squamous carcinoma case $(n=1)$.

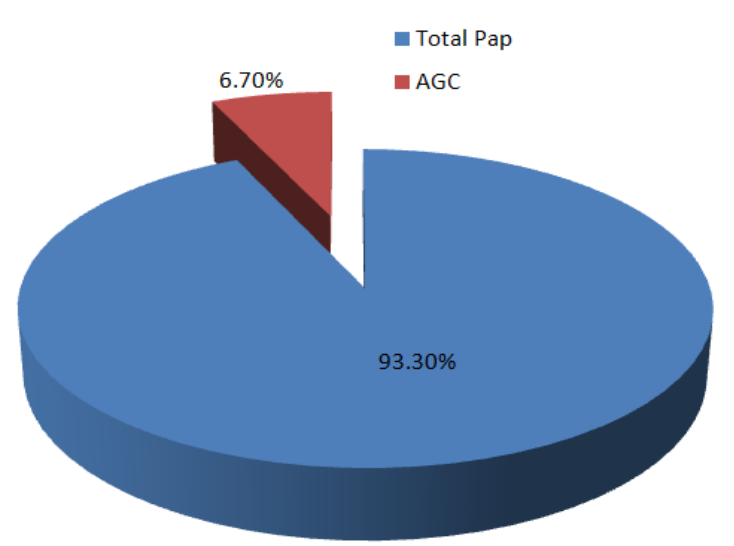

Figure 1. Frequency of atypical glandular cells of undetermined significance $(A G C)$ out of all pregnant and postpartum women Pap smears.

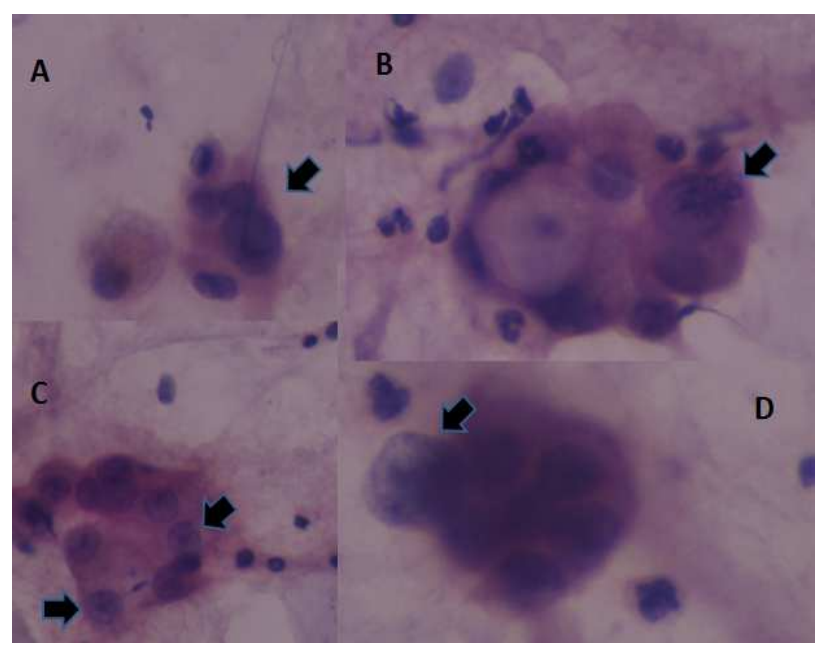

Figure 2. Groups of endocervical cells with abundant cytoplasm and, A: Nuclear enlargement, but uniform, bland looking chromatin (arrow), B: Enlarged cells with distinct cytoplasmic vacuoles. Some cells show a thick nuclear membrane and slightly coarse chromatin (arrow), C: Prominent nucleoli (arrow), D: Variably sized, crowded and hyperchromatic cells; one cell is invariably enlarged with a coarse and somewhat cleared chromatin (arrow) (H\&E stain, x400).

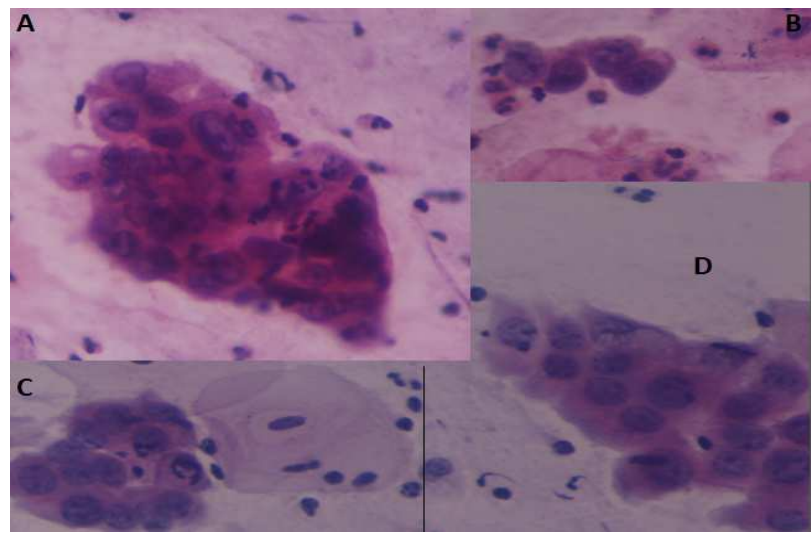

Figure 3. A. group of endocervical cells exhibiting atypical squamous metaplasia, the nuclear membranes are thickened and moderately irregular. Nuclei are round to oval with course, somewhat cleared chromatin and prominent nucleoli (ASC-US/AGUS). B-D: Glandular cells showing enlarged nuclei with smooth nuclear membranes and cleared chromatin (H\&E stain, x400).

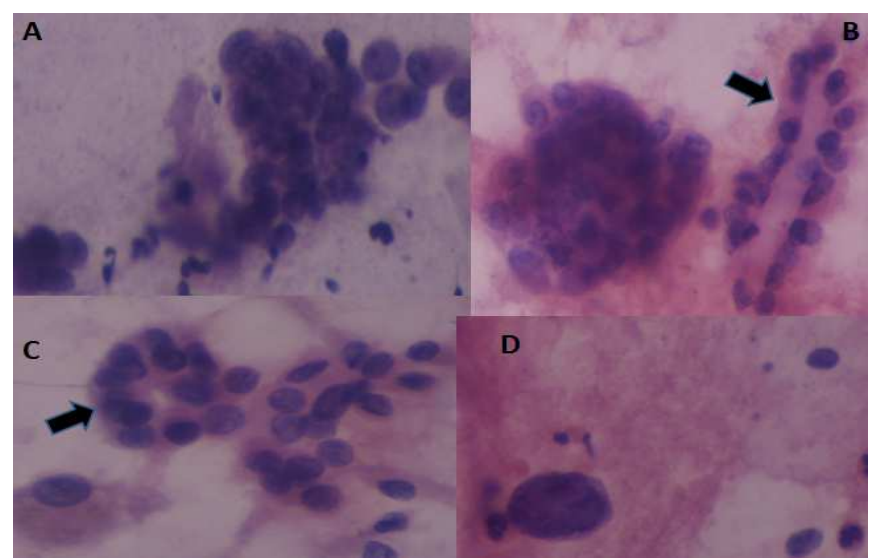

Figure 4. Atypical endometrial cells- A: Architecture is disrupted with nuclear crowding, and "molding". B: Cells still maintain the glandular configuration, but there is evidence of prominent nucleoli with formation of pseudo-papillae (arrow). C: A tendency to form pseudo-rosettes (arrow). D: Bared nucleus with increased N/C ratio, irregular nuclear membrane and hyperchromatism (H\&E stain, x400).

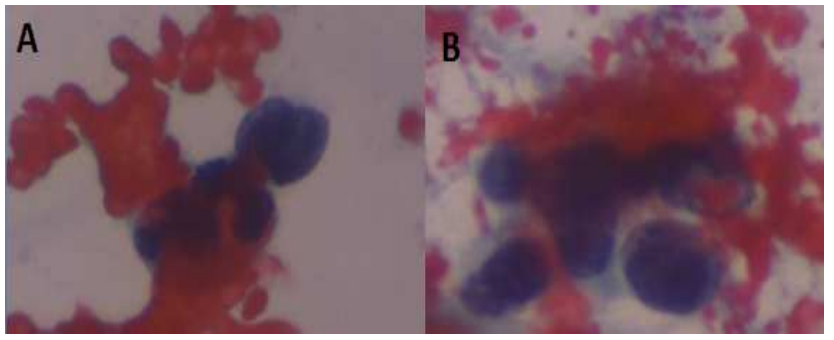

Figure 5 (A-B). Atypical endometrial cells that were proved to be endometrial adenocarcinoma on subsequent follow up (Pap stain 400$)$.

The overall proportion of HR-HPV DNA amplicons in patients at risk (AGC and premalignant) was 22.9\%. Squamous carcinoma was positive while adenocarcinoma was negative for the viral DNA. All tested polyps, microglandular hyperplasia and NILM cases were also negative "Fig. 6". HPV positivity was significantly higher among both AGC and premalignant groups when compared with NILM and non-neoplastic groups $(\mathrm{P}=0.008)$. As well, a 
significantly higher viral DNA difference was observed among premalignant cases when compared with those showing persistent AGC $(\mathrm{P}=0.04)$. However, the differences between different premalignant and malignant groups didn't reach the level of significance $(\mathrm{P}>0.05)$.

Table 1. Detailed follow up results of AGC Pap smears among pregnant and postpartum women.

\begin{tabular}{|c|c|c|}
\hline Diagnosis & $\begin{array}{c}\text { Number } \\
(\%)\end{array}$ & $\begin{array}{c}\text { Positive HR-HPV } \\
(\%)\end{array}$ \\
\hline NILM & $63(41.5)$ & $0 / 5(0.0)$ \\
\hline AGC & $25(16.5)$ & $1 / 15(6.6)$ \\
\hline \multicolumn{2}{|c|}{ Non-neoplastic $(n=54 ; 35.5 \%)$} & $0 / 14$ \\
\hline Decidua & $14(9.2)$ & - \\
\hline Endocervical polyp & $12(7.9)$ & $0 / 5$ \\
\hline Endometrial polyp & $7(4.6)$ & $0 / 5$ \\
\hline Tophoblasts & $5(3.3)$ & - \\
\hline MGH & $4(2.6)$ & $0 / 4$ \\
\hline \multicolumn{2}{|c|}{ Premalignant lesions $(n=20 ; 11.5 \%) *$} & $7 / 20(35)$ \\
\hline LSIL & $6(3.9)$ & $1 / 6(16.6)$ \\
\hline HSIL & $8(5.2)$ & $5 / 8(62.5)$ \\
\hline AIS & $6(3.9)$ & $1 / 6(16.6)$ \\
\hline Carcinoma & $2(1.3)$ & $1 / 2 * *(50)$ \\
\hline Total & 152 & $9 / 56(16.1)$ \\
\hline
\end{tabular}

* $\mathrm{p}=0.008$ (Fishers Exact test), **: The positive carcinoma case was squamous carcinoma. HR-HPV: High risk human papilloma virus, NILM: Negative for intraepithelial lesion or malignancy, AGC: Atypical glandular cells of undetermined significance, MGH: Microglandular hyperplasia, LSIL: Low squamous intraepithelial lesion, HSIL: High squamous intraepithelial lesion, AIS: Adenocarcinoma in situ.

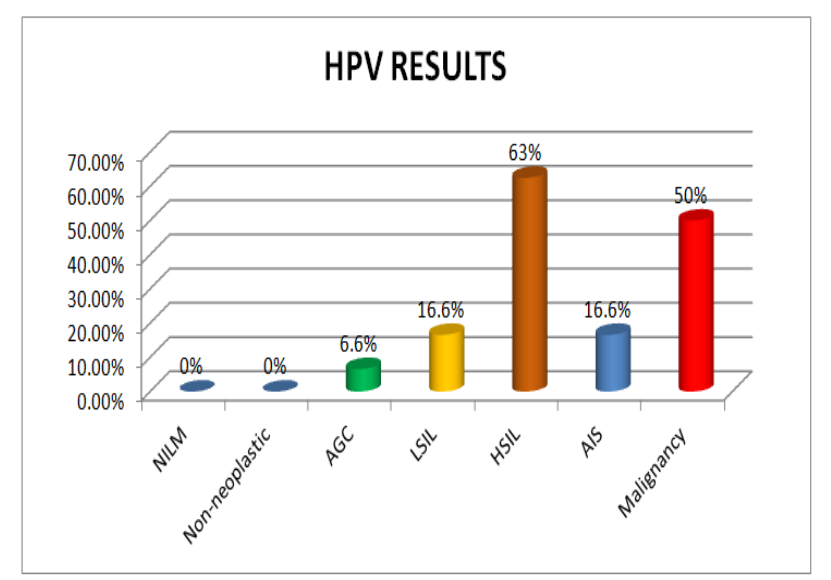

Figure 6. High risk HPV results in the studied cases $(n=56)$. NILM: Negative for intraepithelial lesion or malignancy, AGC: Atypical glandular cells of undetermined significance, LSIL: Low squamous intraepithelial lesion, HSIL: High squamous intraepithelial lesion, AIS: Adenocarcinoma in situ.

\section{Discussion}

Overall, $6.7 \%$ of pregnant and postpartum women had AGC Pap reading. Wide range rates were demonstrated in the literature $(0.08 \%-34.6 \%) .^{5,18,19,20}$ Compared with ours, much lower percentages were observed by Tam et al in Hong Kong population $(0.07 \%)$, Chhieng et al among Birmingham's women $(0.567 \%)$, in a literature review done by Schnatz et al $(0.29 \%)$, Kim et al among Koreans $(0.08 \%)$, Kapila et al on Kuwait population $(0.8 \%)$ and Simavli et al among Turkish people (0.03\%). 3,4,8,18,21,22 Contrarily, much higher ASC-US/AGC rate $(34.6 \%)$ was demonstrated by Chen et al among Taiwanese women. ${ }^{19}$ A concomitant ASC-US reading might account for the latter's' high value. In our series, we reported a concomitant ASC-US in only 7 cases. Despite the fact that could be actual differences, lack of definite morphologic criteria with no consensus in making the diagnosis of AGC among the pathologists, reflected in a high degree of interobserver variation, may account for this wide range. ${ }^{2,19,20}$ The discrepancy might be further contributed to the impact of the employed staining technique. In our clinic we experienced a double staining technique, in the first 2 years of our clinic; we applied H\&E stains followed by the conventional Pap staining technique for the remaining 6 years. This might further influence our results.

It is worth to mention that from the initial AGC cytology in our series, $29.6 \%$ of cases appeared to be at risk of cervical epithelial lesions and required a closer follow up. Comparable results were reported by a meta-analysis done by Schnatz et al, Chhieng et al in Birmingham's population and Kim et al among Koreans (29\%, 29.4\%, 25\% respectively). ${ }^{8,14,18}$

On subsequent follow up, we faced a relatively low histologically confirmed LSIL or more (14.5\%) compared with that observed by Tam et al among Hong Kong pregnant women $(47.5 \%)$ and Chen et al among Taiwanese women $(42.4 \%) .{ }^{3,19}$ Others, however have reported $3 \%$ to $80 \%$ clinically significant cervicouterine lesions on subsequent follow up of women with AGC. $3,5,8,11,19,23-25$ The frequency of clinically significant lesions in women with AGC varies with the population studied, the patient's ages, and women's gestational period examined. $3,4,5,11,12,13,19$

Despite the concept that glandular abnormalities remain the most commonly diagnosed neoplasms in women with AGC Pap smear, 2,5,8,14,18 in the course of this experiment we faced an exciting observation that is SIL cases doubled those of AIS. Perhaps, a concomitant ASC-US influenced our initial AGC reading in these cases.

Another important finding which is worth mentioning is that 2 women had cancer on histologic diagnosis, one was well differentiated endometrial adenocarcinoma, and the second cancer was well differentiated cervical squamous carcinoma. The latter was initially read as ASC-US/AGC. Both cancer cases were proved to be confined to the uterus after hysterectomy (i.e. FIGO I). Despite careful reviewing of the original slides, neither smear showed the typical cytological criteria of invasion such as tumor diathesis, necrosis, single atypical cells nor keratinisation.

Concurrent testing for high-risk HPV is incorporated into the management of women with atypical glandular cells after their initial evaluation with colposcopy. ${ }^{5,8,10,19,26}$ Positive patients for HR-HPV DNA have an increased incidence 
of cervical neoplasia rather than endometrial cancer. ${ }^{26,27}$ As well, HPV test might help in predicting the outcome of pregnant and postpartum women with AGC cytology. ${ }^{8}$ In our clinic, HR-HPV DNA amplicons were positive in $16.1 \%$ of the tested women. Others, however, have shown that $24 \%$ to $43 \%$ of cases test positive for HR-HPV DNA. ${ }^{26-29}$ The explanation for these variable range rates is multifactorial, probably the most important is the use of non-standardized assay methods for detection of viral amplicons and the application of different interpretation criteria for analyzing the resultant findings. Other factors include differences in the populations studied (whether risky area or not), patient's age, HPV status and the viral load. ${ }^{4,19,26-29}$ In the ongoing study, HPV positivity was significantly higher among the women at risk (AGC and premalignant) as compared to NILM and non-neoplastic groups. As well, the viral DNA was significantly higher among premalignant cases when compared with those showing persistent AGC. Comparable findings were observed by Schnatz et al and Chen eta al. ${ }^{8,19}$ However, the viral load difference between SIL groups and invasive malignancy was statistically not significant. Hence, HPV testing might not of help in selecting women who have premalignant lesions from those of invasive malignancy, perhaps due to the similar prevalence rates of viral DNA among both groups.

\section{Conclusions}

Our findings reinforce the experience of others that pregnant and postpartum women with AGC cytology, identical to non-pregnant, are at risk of harboring preneoplastic or neoplastic (squamous or glandular) lesions; testing of AGC cytologic specimens for HR-HPV might help predict neoplastic cases but not to distinguish malignant from premalignant lesions.

\section{References}

[1] TC Jr Wright, JT Cox, LS Massad, LB Twiggs, EJ Wilkinson. 2001 consensus guidelines for the management of women with cervical cytological abnormalities. JAMA. 2002;287:2120-9.

[2] D Solomon, D Davey, R Kurman. The 2001 Bethesda System: terminology for reporting results of cervical cytology. JAMA 2002;287:2114-9.

[3] KF Tam, AN Cheung, E Szeto, HY Ngan. Atypical glandular cells diagnosed during pregnancy and the postpartum period: a retrospective analysis. $2011 \mathrm{Apr} ; 155(2): 213-6$.

[4] DC Chhieng, S Gallaspy, H Yang, J Roberson, I Eltoum. Women with atypical glandular cells. A long-term follow-up study in a high-risk population. Am J Clin Pathol. 2004;122:575-9.

[5] TC Jr Wright, LS Massad, CJ Dunton, M Spitzer, EJ Wilkinson, D Solomon; 2006 ASCCP-Sponsored Consensus Conference. 2006 consensus guidelines for the management of women with abnormal cervical screening tests. J Low Genit Tract Dis. 2007;11(4):201-22.
[6] MK Frey, D Gupta. Evaluation of Women with Atypical Glandular Cells on Cervical Cytology. Gynecologic oncology. 2011;36(9):23-29.

[7] CV Gonçalves, G Duarte, JSD da Costa, AC Marcolin, MS Bianchi VMS, D Dias, et al. Diagnosis and treatment of cervical cancer during pregnancy. Sao Paulo Med J. 2009;127(6):359-65.

[8] PF Schnatz, KE Sharpless, DM O'Sullivan. Use of human papillomavirus testing in the management of atypical glandular cells. J Low Genit Tract Dis. 2009;13(2):94-101.

[9] J Slama, P Freitag, P Dundr, J Duskova, D Fischerova, M Zikan, et al. Outcomes of pregnant patients with Pap smears classified as atypical glandular cells. Cytopathology. 2012;23(6):383-8.

[10] IS Pity, MY Shamdeen, SA Wais. Follow up of Atypical Squamous Cell Pap Smears in Iraqi Women. APJC. 2012;13:3455-60.

[11] C Zhao, RM Austin, J Pan, N Barr, SE Martin, A Raza, C Cobb. Clinical significance of atypical glandular cells in conventional Pap smears in a large, high-risk U.S. West Coast minority population. Acta Cytol. 2009;53(2):153-9.

[12] PP Koonings, JH Price. Evaluation of atypical glandular cells of undetermined significance: is age important? Am J Obstet Gynecol. 2001;184(7):1457-9.

[13] KE Sharpless, PF Schnatz, S Mandavilli, JF Greene, JI Sorosky. Dysplasia associated with atypical glandular cells on cervical cytology. Obstet Gynecol. 2005;105(3):494-500.

[14] DC Chhieng, P Elgert, JF Cangiarella, JM Cohen. Significance of AGUS Pap smears in pregnant and postpartum women. Acta Cytol. 2001;45(3):294-9.

[15] S Sarkar, S Yusif, D Egan. Cervical screening during pregnancy. Ir Med J. 2006;99(9):284-5.

[16] A de-Roda Husman, JMM Walboomers, AJC van den Brule, CJLM Meijer, PJF Snijder. The use of general primers GP5 and GP6 elongated at their 3' ends with adjacent highly conserved sequences improves human papillomavirus detection by PCR. Journal of General Virology. 1995;76:1057-62.

[17] MV Jacobs, PJ Snijders, AJ van den Brule, TJ Helmerhorst, CJ Meijer, JM Walboomers. A general primer GP5+/GP6(+)-mediated PCR-enzyme immunoassay method for rapid detection of 14 high-risk and 6 low-risk human papillomavirus genotypes in cervical scrapings. J Clin Microbiol. 1997;35:791-5.

[18] TJ Kim, HS Kim, CT Park, IS Park, SR Hong, JS Park, et al. Clinical evaluation of follow-up methods and results of atypical glandular cells of undetermined significance (AGUS) detected on cervicovaginal Pap smears. Gynecologic Oncology. 1999;73(2):292-8.

[19] CA Chen, CY Liu, HH Chou, CY Chou, CM Hok, NF Twu, et al. The distribution and differential risks of human papillomavirus genotypes in cervical preinvasive lesions: a Taiwan cooperative oncologic group study. Int J Gynecol Cancer. 2006;16:1801-8.

[20] D Ajit, S Gavas, S Joseph, B Rekhi, K Deodhar, S Kane. Identification of atypical glandular cells in Pap smears: Is it a hit and miss Scenario? Acta Cytologica. 2013;57:45-53. 
[21] K Kapila, SS George, A Al-Shaheen, MS Al-Ottibi, SK Pathan, ZA Sheikh, et al. Changing spectrum of squamous cell abnormalities observed on papanicolaou smears in Mubarak Al-Kabeer Hospital, Kuwait, over a 13-year period. Med Princ Pract. 2006;15(4):253-9.

[22] S Simavli, I Kaygusuz, S Cukur, F Aksel. Abnormal cervical cytology risk factors in the Western Black Sea Region and the importance of health insurance..TurkPatolojiDerg. 2013;24. doi: 10.5146/tjpath.2013.01193

[23] C Wensveen, M Kagie, R Veldhuizen, C De Groot, L Denny, $\mathrm{K}$ Zwinderman, et al. Detection of cervical intraepithelial neoplasia in women with atypical squamous or glandular cells of undetermined significance cytology: a prospective study. Acta Obstet Gynecol Scand. 2003 Sep;82(9):883-9.

[24] AJ Meath, ME Carley, TO Wilson. Atypical glandular cells of undetermined significance. Review of final histologic diagnoses. J Reprod Med. 2002;47(4):249-52.

[25] CS Geier, M Wilson, W Creasman. Clinical evaluation of atypical glandular cells of undetermined significance. Am J Obstet Gynecol. 2001;184(2):64-9.
[26] SF Derchain, SH Rabelo-Santos, LO Sarian, LC Zeferino, ER de Oliveira Zambeli, MC do Amaral Westin, et al. Human papillomavirus DNA detection and histological findings in women referred for atypical glandular cells or adenocarcinoma in situ in their Pap smears. Gynecol Oncol. 2004;95(3):618-23.

[27] C Zhao, A Florea, RM Austin. Clinical utility of adjunctive high-risk human papillomavirus DNA testing in women with Papanicolaou test findings of atypical glandular cells. Arch Pathol Lab Med. 2010;134(1):103-8.

[28] SH Rabelo-Santos, SF Derchain, LL Villa, MC Costa, LO Sarian, MC do Amaral Westin, et al. Human papillomavirus-specific genotypes in cervical lesions of women referred for smears with atypical glandular cells or adenocarcinoma in situ. Int J Gynecol Pathol. 2009;28(3):272-8.

[29] SB Namugenyi, MJ Balsan, SN Glick, JA Jordan. Prevalence and genotype distribution of human papillomavirus in cytology specimens containing atypical glandular cells: A case-control study. J Clin Virol. 2013 Oct;58(2):432-6. 